\title{
Three electrodes touch-mode capacitive pressure sensor
}

\author{
K-M. Chang, G-J. Hwang, Y-L. Hsien
}

\begin{abstract}
A three-electrode capacitive pressure sensor for touch-mode operation with sensitivity of $0.030 \mathrm{pF} / \mathrm{kPa}$ (or $10.4 \mathrm{mV} / \mathrm{kPa}$ using $\mathrm{CP}-10 \mathrm{C} / \mathrm{V}$ converter circuit) in the pressure range of $170-280 \mathrm{kPa}$ is presented with theoretical explanation of experimental results. A special ring structure is designed to integrate a sensing capacitor and a reference capacitor into the same cavity to partially cancel out the temperature effect. A third electrode is included to eliminate two-level connections without reducing its pressure sensitivity. The sensor offers the advantages of simple fabrication processes, planar connections, as well as high sensitivity, near-linear output, and large over-range pressure.
\end{abstract}

\section{1}

\section{Introduction}

The usual capacitive sensors are parallel plate capacitors composed of silicon diaphragm and metal electrode on the insulating substrate. When a pressure is applied, the diaphragm deforms and the sensor capacitance will change accordingly. The capacitance-to-pressure characteristics of the sensor are nonlinear due to the inverse dependence of capacitance on the gap separation [Hanneborg and Ohlckers (1990)]. Specific compensation circuits and modifications to the sensor structure have been applied to reduce the nonlinearity [Puers et al. (1990), Rosengren et al. (1992)]. However, these approaches increase the complexity in either the circuitry or in fabrication processes. A simple and effective method that can be applied to the conventional capacitive sensors to improve linearity is to allow the diaphragm to touch the bottom-insulated electrode [Ding et al. (1990), Ko et al. (1996)]. The increased linearity after touch point pressure is supposed to be due to the nonlinearity of the pressure-capacitance relation being compensated by the membrane becoming more rigid [Rosengren et al. (1992)].

In the literature [Pons et al. (1993), Ko et al. (1982), Schnatz et al. (1992)], two capacitors on the same sensor assembly are necessary for capacitive sensor detecting circuits in order to

K-M. Chang, G-J. Hwang, Y-L. Hsien

Department of Electronics Engineering, National Chiao Tung

University, 1001, Ta Shueh Road, Hsinchu, Taiwan ROC

The authors would like to acknowledge Dr. Wen H. Ko at Case Western Reserve University for his technical consultation. This work is supported by National Science Council under grant NSC 86-2221-E-0090038. compensate the environmental changes and to achieve differential measurement. In general, this approach will increase the sensor size for the dual capacitor design and need additional connections between the electrodes of sensing and reference capacitors. The two-level connections between the electrodes of sensing and reference capacitors. The two-level connections for top and bottom electrodes could be avoided by splitting the capacitors into two parts and connecting in series by the metallization on the plate [Schnatz et al. (1992)]. This arrangement will reduce the capacitance value due to the series connection and hence decrease the sensor sensitivity. In this paper, as shown in Fig. 1, we will present a capacitive pressure sensor for touch mode operation with a special structure design that will allow the integration of two capacitors in a sensor cell without decreasing the sensor sensitivity for planar connections.

\section{2}

\section{Sensor design}

For the design of touch-mode pressure sensor, a computer simulation program has been developed at Case Western Reserve University to accurately simulate the performance of touch mode sensors using the finite element analysis with modified boundary conditions [Ko et al. (1996)]. Their results are used as a reference in our sensor design.

Their simulation curve of normalized deflection ratio $\left(w_{0} / h\right)$ against the normalized pressure $\left(\mathrm{Pa}^{4} / E h^{4}\right)$ for square diaphragm can be used for touch point pressure estimation, where $w_{0}$ is the center deflection of diaphragm; $h$, the diaphragm thickness; $P$, the differential pressure; $a$, the length of square diaphragm; $E$, the Young's modulus. The touch point pressure $P_{0}$ is determined by letting the center deflection $w_{0}$ equal the cavity gap $g$. The touch mode pressure sensor is designed to operate in the linear region $\left(1.2 P_{0}\right.$ to $\left.2 P_{0}\right)$ after touch-point pressure $P_{0}$. A sensor with touch-point pressure of $0.4 \mathrm{~atm}$ differential pressure, between the applied and cavity pressure, can be obtained as the diaphragm parameters, $h=5 \mu \mathrm{m}, a=700 \mu \mathrm{m}$, and $g=3 \mu \mathrm{m}$ are provided.

According to the simulation results for near-linear operation, the maximum touched length of the diaphragm, at full-scale pressure, is about $70 \%$ of the total length of the diaphragm, at full-scale pressure, is about $70 \%$ of the total length of the diaphragm. This implies that the touched area of the sensor under full-scale pressure is about $49 \%$ of the diaphragm area. Therefore, two capacitors $\left(C_{\mathrm{ap}}\right.$ and $C_{\mathrm{bp}}$ defined below) can be placed by a ring structure in one cavity as shown in Fig. 1. The central area is used as the sensing capacitor and 


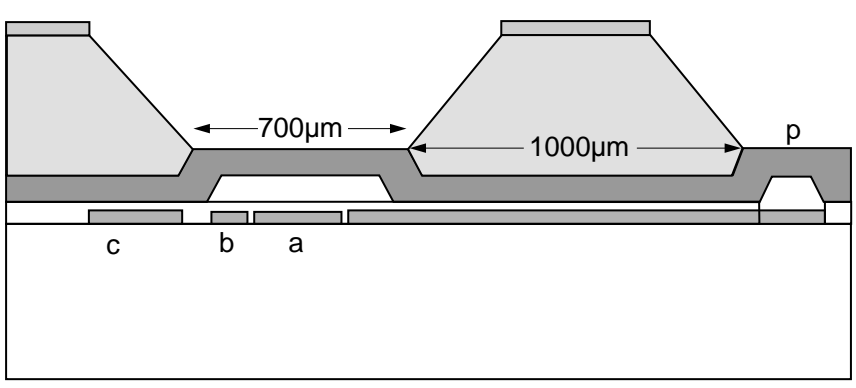

a

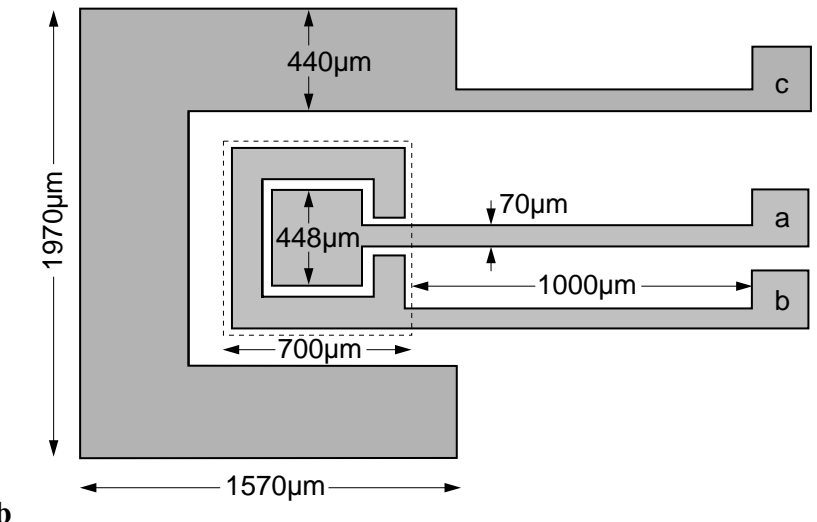

Fig. 1a,b. A schematic drawing of the three electrodes capacitive pressure sensor: a cross-section view $\mathbf{b}$ top view

the edge area as the reference capacitor. Normally, the electrode contact on $\mathrm{p}^{+}$silicon diaphragm is needed. The design of our sensor avoids the need of contacting the electrode on the silicon diaphragm by connecting the capacitors in the cavity in series with a large capacitor $\left(C_{\mathrm{pc}}\right)$ located at the edge under the bonding area. As can be found in Fig. 1, the capacitor $C_{\mathrm{ac}}$ is the series connection of $C_{\mathrm{ap}}$ and $C_{\mathrm{pc}}$ and the $C_{\mathrm{bc}}$ is the series connection of $C_{\mathrm{bp}}$ and $C_{\mathrm{pc}}$. The electrode connections for $C_{\mathrm{ac}}$ and $C_{\mathrm{bc}}$ are planar connections. The large capacitor $C_{\mathrm{pc}}$ is composed by the $\mathrm{p}^{+}$silicon plate and the metal plate on the Pyrex substrate with a thin glass layer that is also used as the insulating layer in the cavity. The large value in capacitance is owing to the high dielectric constant of glass, thin glass spacing and large size.

\section{3}

\section{Fabrication}

The sensors are fabricated using bulk micromachining technique with four masks. The fabrication sequence, illustrated in Fig. 2 at key stages, involves both silicon and glass processing.

$\mathrm{N}$-type, $\left(\begin{array}{lll}1 & 0 & 0\end{array}\right)$ oriented, double-side polished, 4-in silicon wafer with thickness $480 \mu \mathrm{m}$ is used as top wafer. After initial cleaning, a $1 \mu \mathrm{m}$ silicon dioxide is grown by wet oxidation at $1000^{\circ} \mathrm{C}$ for $5 \mathrm{~h}$. The oxide film on the front side of silicon wafer is patterned by standard photolithography and wet-etched in BHF to open window for the cavity formation. The wafer is then immersed in a $33 \mathrm{wt} \% \mathrm{KOH}$ solution at $60^{\circ} \mathrm{C}$ for $10 \mathrm{~min}$ to form cavities of thickness $3 \mu \mathrm{m}$ in depth (Fig. 2a). After cavity formation, the oxide film of the front side is etched off and the
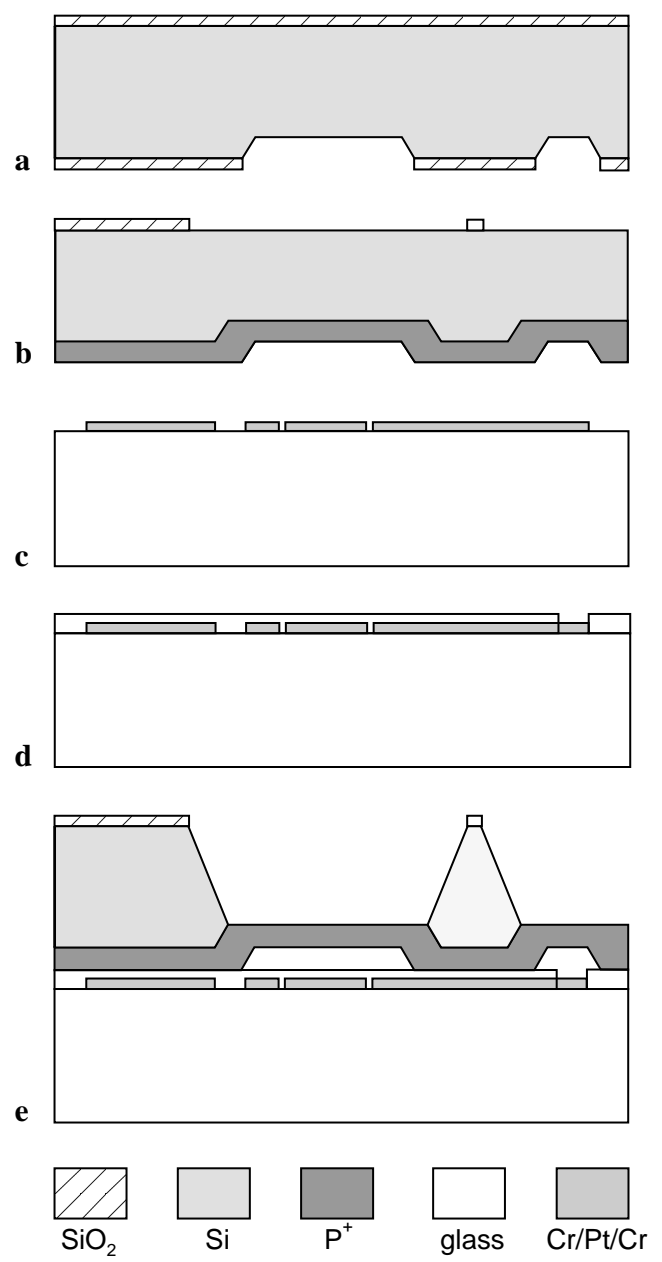

Fig. 2a-e. The fabrication sequence at key stages

oxide film on the backside is protected by photoresist during oxide etching. This oxide film of the backside is used as a mask for the boron diffusion. Next, boron diffusion is carried out at $1125^{\circ} \mathrm{C}$ in a gas mixture of $98 \% \mathrm{~N}_{2}$ and $2 \% \mathrm{O}_{2}$ for $6 \mathrm{~h}$ with boron nitride disk as the diffusion source. A $5 \mu \mathrm{m} \mathrm{p}{ }^{+}$layer is formed on the front side of the silicon wafer [Chang et al. (1998)]. After boron diffusion, steam oxidation is performed at $800^{\circ} \mathrm{C}$ for $30 \mathrm{~min}$ in order to remove the boron silicate glass (BSG) formed during diffusion. The BSG film and the backside oxide film are then patterned and etched to open the windows for the diaphragm formation (Fig. 2b).

Double-side polished, 4-in Pyrex 7740 glass wafer with thickness $500 \mu \mathrm{m}$ is used as the bottom wafer. The lift-off technique is used in the glass processing. After glass cleaning, a stencil layer of photoresist is patterned on the glass wafer. A multi-metal film ( $5 \mathrm{~nm} \mathrm{Cr} / 250 \mathrm{~nm} \mathrm{Pt} / 5 \mathrm{~nm} \mathrm{Cr}$ ) is deposited on the inverted pattern of the stencil layer and the exposed area by electron beam evaporation. After evaporation, a mixture of $\mathrm{H}_{2} \mathrm{SO}_{4}$ and $\mathrm{H}_{2} \mathrm{O}_{2}$ is used to lift-off the stencil layer. The multi-metal film with the desired pattern is left on the glass wafer and forms the bottom plate of the capacitor and the output feedthrough (Fig. 2c). This multi-metal film offers several advantages of reliable interconnect materials with low resistance, good adhesion to the glass, inert reaction to silicon 
etchant $\mathrm{KOH}$ and oxide etchant. The sheet resistance of this multi-metal film is measured to be $20 \Omega$ per square. A glass film of Pyrex 7740 with thickness $0.6 \mu \mathrm{m}$ is then sputtered on the top of the multi-metal diaphragm touched the bottom plate of the metal film and dielectric material of capacitors. After glass sputtering, the contact windows of bonding pads are opened by standard photolithography and wet etching (Fig. 2d).

The prepared top silicon wafer and the glass wafer are aligned and then anodically bonded together. These bonded pair of silicon and glass wafers is then immersed into a $20 \mathrm{wt} \%$ $\mathrm{KOH}$ at $60^{\circ} \mathrm{C}$, in considerations of $\mathrm{Si}$ /oxide selectivity and etch rate, and is etched from the backside of silicon to form the diaphragm (Fig. 2e) [Seidel et al. (1990)]. After completion of backside etching, the wafer is diced into individual chip for pressure testing.

\section{Testing system}

The layout of the test system is shown in Fig. 3. It includes a compressed air tank, a pressure control system, a mechanical pump, a high-pressure chamber, and a capacitance measurement system. The capacitance measurement system includes two measurement units: a capacitance bridge and a CP-10 circuit. A switch is used to select the measurement unit. The CP-10 circuit is a capacitance-to-voltage converter using switched capacitor circuit [Ko and Yeh (1994)]. The block diagram of the CP-10 is shown in Fig. 4. It consists of a voltage, capacitance, and frequency controlled current source (VCFcI), a charge substrator $(\Sigma)$, and a current-to-voltage converter (R). When $C_{\mathrm{f}} \cdot f \cdot R \gg 1$, the output voltage $V_{\text {out }}$ could be determined as

$V_{\text {out }}=\left[\left(V_{\mathrm{g}} \cdot C_{\mathrm{x}}\right)-\left(V_{\mathrm{o}} \cdot C_{\mathrm{o}}\right)\right] / C_{\mathrm{f}}$

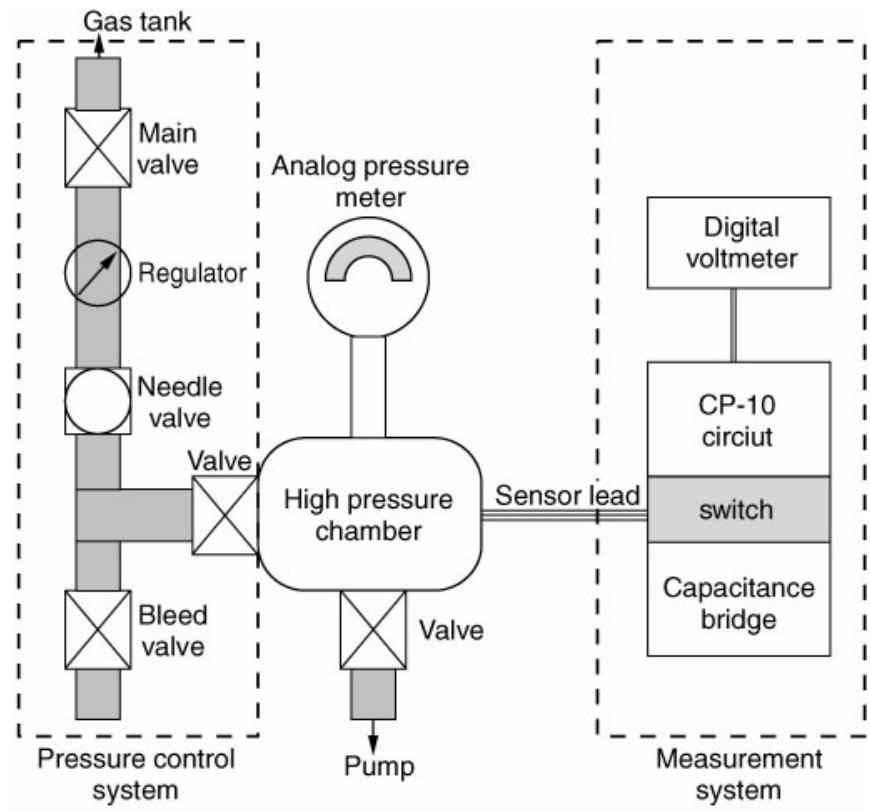

Fig. 3. The layout of test system

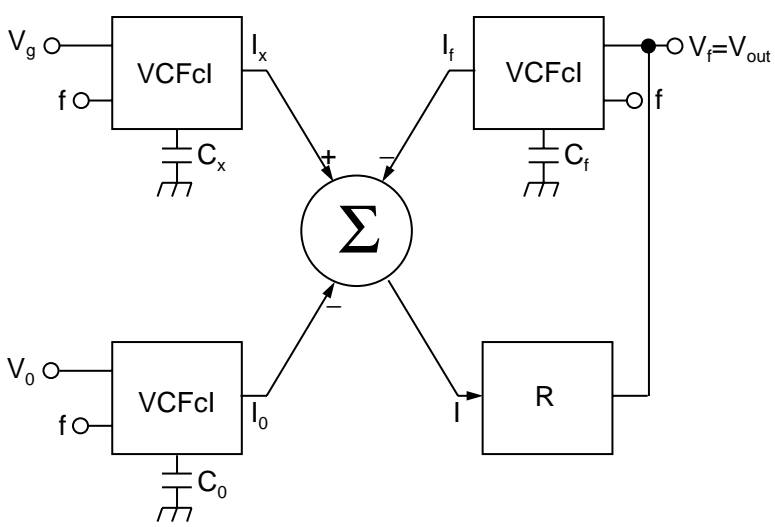

Fig. 4. The block diagram of the CP-10 converter circuit [after Ko (1994)]

where $V_{\mathrm{g}}$ and $V_{\mathrm{o}}$ are the adjustable voltages, $C_{\mathrm{x}}$ is the sensor capacitance, $C_{\mathrm{o}}$ the total internal and external offset capacitor, and $C_{\mathrm{f}}$ the external feedback capacitor. The circuit sensitivity, $S_{\mathrm{c}}$, can be defined as

$S_{\mathrm{c}}=\frac{\mathrm{d} V_{\text {out }}}{\mathrm{d} C_{\mathrm{x}}}=\frac{V_{\mathrm{g}}}{C_{\mathrm{f}}}$

This implies that the circuit sensitivity $S_{\mathrm{c}}$ can be controlled by varying $V_{\mathrm{g}}$ or $C_{\mathrm{f}}$ and is not affected by the stray capacitance. Besides, the offset can be adjusted by changing $V_{\mathrm{o}}$ or $C_{\mathrm{o}}$ without affecting the sensitivity. The sensor fabricated has three terminals on the glass substrate and one connection on the $\mathrm{p}^{+}$diaphragm. Referring to Figs. 1 and 4, the electrode " $\mathrm{a}$ " is for the sensing capacitor $C_{\mathrm{x}}$, electrode " $\mathrm{b}$ " is for the reference capacitor $C_{\mathrm{o}}$ and the electrode " $\mathrm{c}$ " is connected to the ground. If one sensing capacitor $C_{\mathrm{x}}$ on the sensor is used, a conventional external capacitor will be used as $C_{\mathrm{o}}$ for offset adjustment. Otherwise, the central capacitor is used as the sensing capacitor $C_{\mathrm{x}}$ and the edge reference capacitor is used as $C_{\mathrm{o}}$ for offset adjustment.

\section{5}

\section{Results and discussion}

In order to verify the operation of the ring structure of the sensor, an electrical contact on the $\mathrm{p}^{+}$silicon layer is connected. Five capacitance outputs of the capacitors $C_{\mathrm{ap}}, C_{\mathrm{bp}}$, $C_{\mathrm{ac}}, C_{\mathrm{bc}}$ and $C_{\mathrm{ab}}$ are measured by the Capacitance Bridge. These capacitances are defined as follows:

$C_{\text {ap }}$ the capacitance between the central sensing plate on the glass denoted by " $\mathrm{a}$ " and the silicon $\mathrm{p}^{+}$plate denoted by "p"

$C_{\mathrm{bp}}$ the capacitance between the edge reference plate on the glass denoted by " $b$ " and the silicon $\mathrm{p}^{+}$plate

$C_{\mathrm{ac}}$ the capacitance between sensing " $\mathrm{a}$ " and " $\mathrm{c}$ " electrodes. It is the series capacitance of the sensing capacitance $C_{\mathrm{ap}}$ and the large capacitance $C_{\mathrm{pc}}$ (the large capacitance between the silicon $\mathrm{p}^{+}$plate and the metal plate at the clamped area on the glass denoted by " $c$ ")

$C_{\mathrm{bc}}$ the capacitance between reference " $\mathrm{b}$ " and " $c$ " electrodes. It is the series capacitance of the reference capacitance $C_{\mathrm{bp}}$ and the large capacitance $C_{\mathrm{pc}}$ 
$C_{\mathrm{ab}}$ the capacitance of the sensing electrode " $\mathrm{a}$ " and the reference electrode " $\mathrm{b}$ ". It is the series capacitance of $C_{\mathrm{ap}}$ and $C_{\mathrm{bp}}$ in parallel with the parasitic capacitance between "a" and "b" electrodes on the glass substrate $C_{\mathrm{abs}}$

The connections of $C_{\mathrm{ap}}$ and $C_{\mathrm{bp}}$ are the two-level connections and those of $C_{\mathrm{ac}}, C_{\mathrm{bc}}$, and $C_{\mathrm{ab}}$ are the planar connections.

Four voltage outputs, namely $V_{\text {ab-p }}, V_{\text {ab-c }}, V_{\text {ap }}$, and $V_{\text {ac }}$, are also measured by the $\mathrm{CP}-10$ circuit. These voltage outputs are defined as follows:

$V_{\text {ab-p }}$ the output voltage from CP-10 as connecting the $C_{\text {ap }}$ as the $C_{\mathrm{x}}$ and the $C_{\mathrm{bp}}$ as the $C_{\mathrm{o}}$ on the CP-10 circuit board

$V_{\mathrm{ab}-\mathrm{c}}$ the output voltage from CP-10 as connecting the $C_{\mathrm{ac}}$ as the $C_{\mathrm{x}}$ and the $C_{\mathrm{bc}}$ as the $C_{\mathrm{o}}$

$V_{\text {ap }}$ the output voltage from CP-10 as connecting the $C_{\mathrm{ap}}$ as the $C_{\mathrm{x}}$ and a conventional capacitor as the $C_{\mathrm{o}}$.

$V_{\mathrm{ac}}$ the output voltage form CP-10 as connecting the $C_{\mathrm{ac}}$ as the $C_{\mathrm{x}}$ and a conventional capacitor as the $C_{\mathrm{o}}$

\section{1}

\section{Capacitance output}

Figure 5 presents the measured capacitance-to-pressure characteristics of the five capacitors at $25^{\circ} \mathrm{C}$. The pressure inside the sensor cavity is 1 atm $(101 \mathrm{kPa})$ because it sealed at 1 atm and $25^{\circ} \mathrm{C}$. As shown in Fig. 5, the measured capacitance $C_{\text {ap }}$ at zero differential pressure between the applied and cavity pressure is $12.249 \mathrm{pF}$. This measured capacitance $12.249 \mathrm{pF}$ is larger than the calculated capacitance $0.6 \mathrm{pF}$ for the center capacitor inside the cavity with electrode area $448 \mu \mathrm{m} \times$ $448 \mu \mathrm{m}$, cavity gap $3 \mathrm{e} \mu \mathrm{m}$, and air dielectric constant. The difference between 12.249 and $0.6 \mathrm{pF}$ is due to the existence of two capacitances that are connected in parallel with the capacitors inside the cavity. The first one is the capacitance of the metal film feedthrough located at the bonding area from

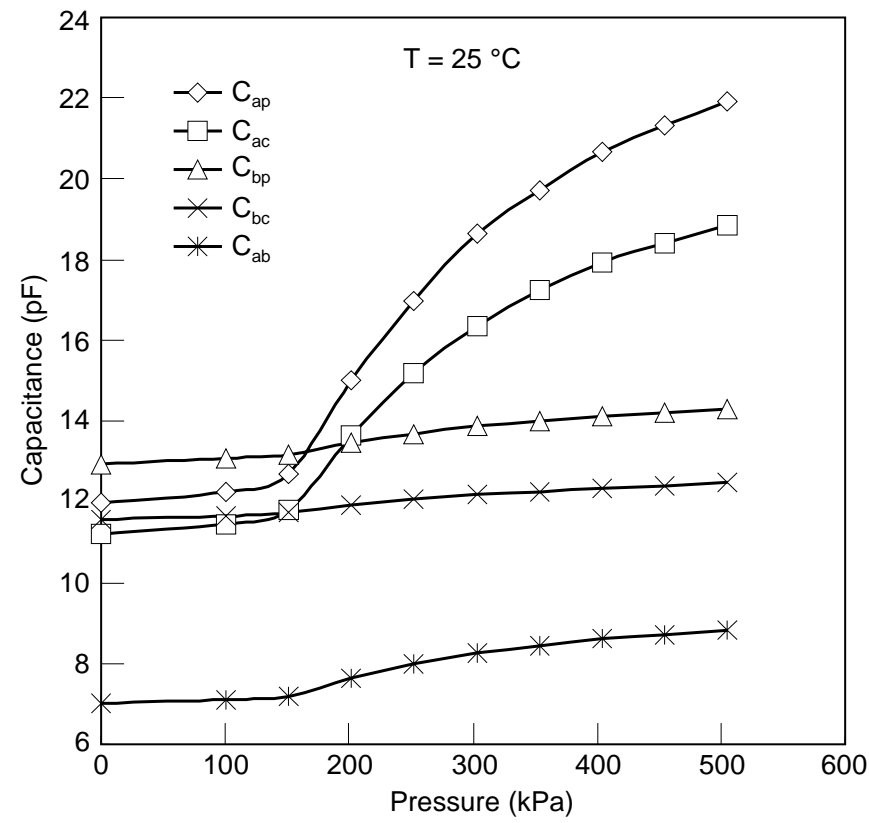

Fig. 5. The capacitance-to-pressure characteristics of the sensor the electrode inside cavity to the contact pad. The other is the stray capacitance of test system from the sensor contact pad to the Capacitance Bridge in the test system. The metal film feedthrough capacitance is estimated to be $5.5 \mathrm{pF}$ and the stray capacitance of the test system is measured to be $6 \mathrm{pF}$. The metal film feedthrough with area of length $1000 \mu \mathrm{m}$ and width $70 \mu \mathrm{m}$, as shown in Fig. $1 \mathrm{~b}$, and the $\mathrm{p}^{+}$silicon layer with a $0.6 \mu \mathrm{m}$ glass layer between them form a capacitor that is connected in parallel with the capacitors inside the cavity. The $1000 \mu \mathrm{m}$ length of metal film feedthrough is needed for a silicon wafer with thickness $480 \mu \mathrm{m}$ etched in $\mathrm{KOH}$ for the openings of the diaphragm and contact pads. This metal film feedthrough capacitor is similar to the capacitor $C_{\mathrm{pc}}$ under the bonding area but has small area. According to the sensor design, the area of $C_{\mathrm{pc}}$ is 24 times the area of metal film feedthrough capacitor. The measured capacitance of $C_{\mathrm{pc}}$ is $140 \mathrm{pF}$ including the stray capacitance of test system $6 \mathrm{pF}$. This gives that the capacitance of metal film feedthrough is $5.5 \mathrm{pF}$ $(\approx 134 / 24)$. The area of capacitor $C_{\mathrm{bp}}$ inside cavity is designed to be nearly equal to, but slightly larger than, the area of the capacitor $C_{\mathrm{ap}}$. Therefore, the measured capacitance of $C_{\mathrm{bp}}$ at zero differential pressure is $13.069 \mathrm{pF}$ as illustrated in Fig. 5. The capacitance of $C_{\mathrm{ac}}$ is the series capacitance of the central sensing capacitance $C_{\mathrm{ap}}$ and the large capacitance $C_{\mathrm{pc}}$ as

$C_{\mathrm{ac}}=C_{\mathrm{ap}} C_{\mathrm{pc}} /\left(C_{\mathrm{ap}}+C_{\mathrm{pc}}\right)$

This series connection implies that the capacitance $C_{\mathrm{ac}}$ is smaller than the capacitance $C_{\mathrm{ap}}$. The measured capacitance $C_{\mathrm{ac}}$ at zero differential pressure is $11.444 \mathrm{pF}[\approx 12.249 \times$ $140 /(12.249+140)]$, which is smaller than the measured capacitance $C_{\mathrm{ap}}$ of $12.249 \mathrm{pF}$. Accordingly, the capacitance $C_{\mathrm{bc}}$ is the series capacitance of $C_{\mathrm{bp}}$ and $C_{\mathrm{pc}}$ as

$C_{\mathrm{bc}}=C_{\mathrm{bp}} C_{\mathrm{pc}} /\left(C_{\mathrm{bp}}+C_{\mathrm{pc}}\right)$

The measured capacitance $C_{\mathrm{bc}}$ at zero differential pressure is $11.662 \mathrm{pF}[\approx 13.069 \times 140 /(13.069+140)]$ that is smaller than the capacitance $C_{\mathrm{bp}}$ of $13.069 \mathrm{pF}$. Finally, the capacitance of $C_{\mathrm{ab}}$ is the series capacitance of two nearly capacitance $C_{\mathrm{ap}}$ and $C_{\mathrm{bp}}$, plus the parasitic capacitance between " $\mathrm{a}$ " and " $\mathrm{b}$ " electrodes on the glass substrate $C_{\mathrm{abs}}$ as

$C_{\mathrm{ab}}=\left[C_{\mathrm{ap}} C_{\mathrm{bp}} /\left(C_{\mathrm{ap}}+C_{\mathrm{bp}}\right)\right]+C_{\mathrm{abs}}$

The measured capacitance of $C_{\mathrm{ab}}$ is $7.094 \mathrm{pF}$.

The sensor is designed to have differential touch point pressure $0.4 \mathrm{~atm}(40 \mathrm{kPa})$ as discussed in Section 2. The pressure inside the cavity is $1 \mathrm{~atm}(101 \mathrm{kPa})$. As can be found in Fig. 5, the center of the diaphragm touches the bottom of the cavity around $141 \mathrm{kPa}$. After touch point pressure, the values of capacitances $C_{\mathrm{ap}}, C_{\mathrm{ac}}$, and $C_{\mathrm{ab}}$ increase linearly first and then become saturated in the high-pressure range. The outputs of edge reference capacitors $C_{\mathrm{bp}}$ and $C_{\mathrm{bc}}$ are almost unchanged as applied pressure because $C_{\mathrm{bp}}$ is placed outside the deformed area of the diaphragm. In general, these experimental curves are in good agreement with the sensor design for touch-mode operation.

The average pressure sensitivity of the sensor is determined in the linear region from 170 to $280 \mathrm{kPa}$ after touch-point pressure $141 \mathrm{kPa}$ according to the sensor design. As shown in Fig. 5, the average pressure sensitivity of $C_{\mathrm{ac}}\left(\Delta C_{\mathrm{ac}} / \Delta P\right)$ is 
$0.030 \mathrm{pF} / \mathrm{kPa}$ and that of $C_{\mathrm{ap}}\left(\Delta C_{\mathrm{ap}} / \Delta P\right)$ is $0.037 \mathrm{pF} / \mathrm{kPa}$. The value of $\Delta C_{\mathrm{ac}} / \Delta P$ is about $20 \%(=|0.030-0.037| / 0.037)$ less than that of $\Delta C_{\mathrm{ap}} / \Delta P$. This difference is again due to the existence of the metal film feedthrough capacitance and the spray capacitance of the test system as discussed before. According to (5.1) along with the pressure-independent capacitance of $C_{\mathrm{pc}}\left(\Delta C_{\mathrm{pc}} / \Delta P=0\right)$ that is located under the bonding area, $\Delta C_{\mathrm{ac}} / \Delta P$ becomes

$\Delta C_{\mathrm{ac}} / \Delta P=\frac{\Delta C_{\mathrm{ap}} / \Delta P\left(C_{\mathrm{pc}}\left(C_{\mathrm{ap}}+C_{\mathrm{pc}}\right)-C_{\mathrm{ap}} C_{\mathrm{pc}}\right)}{\left(C_{\mathrm{ap}}+C_{\mathrm{pc}}\right)^{2}}$

The capacitance of $C_{\text {ap }}$ at the $225 \mathrm{kPa}$ midpoint-pressure of the operation range from 170 to $280 \mathrm{kPa}$ is $16 \mathrm{pF}$ as shown in Fig. 5. From (5.4) and $C_{\mathrm{pc}}=140 \mathrm{pF}$, we have

$$
\begin{aligned}
\Delta C_{\mathrm{ac}} / \Delta P & =\frac{\Delta C_{\mathrm{ap}} / \Delta P(140 \times(16+140)-16 \times 140)}{(16+140)^{2}} \\
& =0.8 \frac{\Delta C_{\mathrm{ap}}}{\Delta P}
\end{aligned}
$$

This implied that the smaller the metal film feedthrough capacitance and the stray capacitance of test system as compared to $C_{\mathrm{pc}}=140 \mathrm{pF}$, the closer the pressure sensitivity of $C_{\mathrm{ac}}\left(\Delta C_{\mathrm{ac}} / \Delta P\right)$ and $C_{\mathrm{ap}}\left(\Delta C_{\mathrm{ap}} / \Delta P\right)$ will be. This relatively large value of the metal film feedthrough capacitance and stray capacitance of the measuring system is also the cause of the large discrepancy between $C_{\mathrm{ap}}$ and $C_{\mathrm{ac}}$ and the poor linearity in the high-pressure range, as illustrated in Fig. 5.

The pressure sensitivity of $C_{\mathrm{ab}}\left(\Delta C_{\mathrm{ab}} / \Delta P\right)$ is $0.007 \mathrm{pF} / \mathrm{kPa}$, which is less than the quarter value of the pressure sensitivity of $C_{\mathrm{ac}}$ or $C_{\mathrm{ap}}$. This measured result is expected as a consequence of the series connection of $C_{\mathrm{ab}}$. According to the data from Fig. 5, the capacitances of $C_{\mathrm{ap}}$ and $C_{\mathrm{bp}}$ at the $225 \mathrm{kPa}$ midpoint-pressure of the operation range are 16 and $14.5 \mathrm{pF}$, respectively, and the capacitance of $C_{\mathrm{bp}}$ is insensitive to the applied pressure $\left(\Delta C_{\mathrm{bp}} / \Delta P \cong 0\right)$. The parasitic capacitance between " $a$ " and " $b$ " electrodes on the glass substrate $C_{a b s}$ would not be changed significantly with the pressure and could be neglected here. Therefore, $\Delta C_{\mathrm{ab}} / \Delta P$ from (5.3) will become

$$
\begin{aligned}
\Delta C_{\mathrm{ab}} / \Delta P & =\frac{\Delta C_{\mathrm{ap}} / \Delta P\left(C_{\mathrm{bp}}\left(C_{\mathrm{ap}}+C_{\mathrm{bp}}\right)-C_{\mathrm{ap}} C_{\mathrm{bp}}\right)}{\left(C_{\mathrm{ap}}+C_{\mathrm{bp}}\right)^{2}} \\
& =\frac{\Delta C_{\mathrm{ap}} / \Delta P(14.5 \times(16+14.5)-16 \times 14.5)}{(16+14.5)^{2}} \\
& \leqslant \frac{1}{4} \frac{\Delta C_{\mathrm{ap}}}{\Delta P}
\end{aligned}
$$

The structure of using $C_{\mathrm{ab}}$ instead of $C_{\mathrm{ap}}$ as sensing capacitor $C_{\mathrm{x}}$ is to avoid the need for contacting the upper electrode on the $\mathrm{p}^{+}$layer. This is done by splitting the capacitor $C_{\mathrm{x}}$ into two capacitors $C_{\mathrm{ap}}$ and $C_{\mathrm{bp}}$ and connecting in series through the $\mathrm{p}^{+}$ layer [Ko et al. (1982)]. This technique trades the benefit of planar connection at the sacrifice of its sensitivity. However, our special three-electrodes structure using $C_{\mathrm{ac}}$ as sensing capacitor and $C_{\mathrm{bc}}$ as a reference capacitor offers the advantage of planar connection without decreasing sensitivity and differential sensing to balance out common mode environmental inference.

\section{2}

\section{Voltage output of CP-10}

The voltage output of CP-10, $V_{\text {out }}$, defined in Sect. 4 is given as

$V_{\text {out }}=\left[\left(V_{\mathrm{g}} \cdot C_{\mathrm{x}}\right)-\left(V_{\mathrm{o}} \cdot C_{\mathrm{o}}\right)\right] / C_{\mathrm{f}}$

In the $V_{\text {ap }}$ (or $V_{\text {ac }}$ ) measurements, the central sensing capacitor $C_{\mathrm{ap}}\left(\right.$ or $C_{\mathrm{ac}}$ ) is connected to $C_{\mathrm{x}}$ with $\mathrm{p}$ (or c) connected to the ground of CP-10 and a conventional $17 \mathrm{pF}$ capacitor is connected to $C_{\mathrm{o}}$ of CP-10 for offset adjustment. In the $V_{\mathrm{ab}-\mathrm{p}}$ (or $V_{\text {ab-c }}$ ) measurements, the central sensing capacitor $C_{\mathrm{ap}}\left(\right.$ or $C_{\mathrm{ac}}$ ) is connected to $C_{\mathrm{x}}$, the edge reference capacitor $C_{\mathrm{bp}}$ (or $C_{\mathrm{bc}}$ ) is connected to $C_{\mathrm{o}}$ for offset adjustment, and p (or c) is the ground. In this study the offset voltage $V_{\mathrm{o}}$ is set to $2.730 \mathrm{~V}$ and the circuit sensitivity $S_{\mathrm{c}}, V_{\mathrm{g}} / C_{\mathrm{f}}$, is kept to a fixed value by setting $V_{\mathrm{g}}=2.700 \mathrm{~V}$ and $C_{\mathrm{f}} \cong 5 \mathrm{pF}$. The output swing is from 1.3 to $4 \mathrm{~V}$ of $\mathrm{CP}-10$ for a $5 \mathrm{~V}$ power supply.

Figure 6 presents the measured pressure characteristics of output voltages of $V_{\mathrm{ap}}, V_{\mathrm{ac}}, V_{\mathrm{ab}-\mathrm{p}}$, and $V_{\mathrm{ab}-\mathrm{c}}$. The curves of $V_{\text {ap }}$ and $V_{\text {ac }}$ exhibit similar pressure dependence as those of $C_{\text {ap }}$ and $C_{\text {ac }}$ obtained from the capacitance measurements. However, the discrepancy between the $V_{\text {ap }}$ and $V_{\mathrm{ac}}$ in the high-pressure range (over $280 \mathrm{kPa}$ ) in Fig. 6 is smaller than the discrepancy between the $V_{\mathrm{ap}}$ and $V_{\mathrm{ac}}$ in the high-pressure range (over $280 \mathrm{kPa}$ ) in Fig. 6 is smaller than the discrepancy between the $C_{\mathrm{ap}}$ and $C_{\mathrm{ac}}$ in Fig. 5. This is due to the effect that the CP-10 circuit measures the capacitance difference and does not have the sum of all capacitors as denominator in $V_{\text {out }}$ or $S_{\mathrm{c}}$ equation. It could reduce the stray capacitance effect through the offset adjustment of either $V_{\mathrm{o}}$ or $C_{\mathrm{o}}$. Thus, the outputs of $V_{\mathrm{ac}}$ are closer to those of $V_{\text {ap }}$ in the high-pressure range and the pressure sensitivity of $V_{\text {ac }}$ will be close to the pressure sensitivity of $V_{\text {ap }}$ in the operation pressure range. The measured pressure sensitivity of $V_{\mathrm{ap}}\left(\Delta V_{\mathrm{ap}} / \Delta P\right)$ is $13.3 \mathrm{mV} / \mathrm{kPa}$

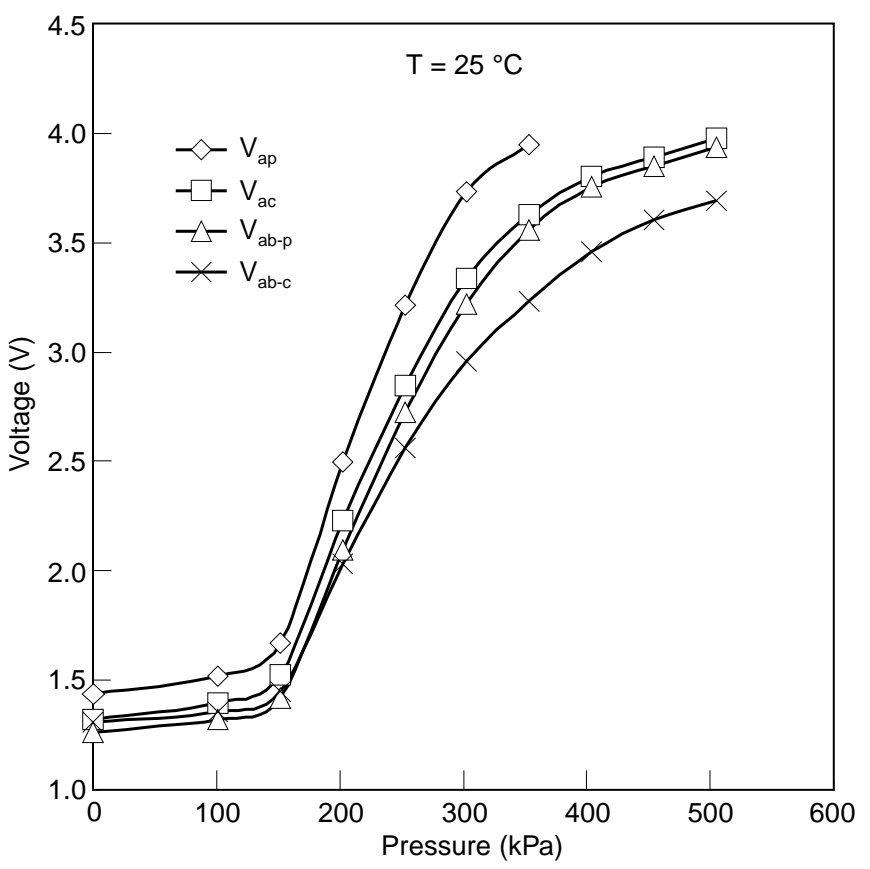

Fig. 6. The voltage-to-pressure characteristics of the sensor 
and the pressure sensitivity of $V_{\mathrm{ac}}\left(\Delta V_{\mathrm{ac}} / \Delta P\right)$ is $11.8 \mathrm{mV} / \mathrm{kPa}$. Their difference is $10 \%$, while the difference between the pressure sensitivity of $C_{\mathrm{ac}}$ and $C_{\mathrm{ap}}$ obtained from the capacitance measurements is $20 \%$. In fact, this $10 \%$ difference in the pressure sensitivity is theoretically accountable by the planar connections of the ring structure for touch mode operation. In the absence of metal film feedthrough capacitance and stray capacitance of measuring system, the capacitance of $C_{\text {ap }}$ in the cavity at $225 \mathrm{kPa}$ would be about $4.5 \mathrm{pF}(=16-(6+5.5))$ and the capacitance of $C_{\mathrm{pc}}$ is $134 \mathrm{pF}$ $(=140-6)$. According to (5.4), the value of $\Delta C_{\mathrm{ac}} / \Delta P$ is about $90 \%$ of the value of $\Delta C_{\mathrm{ap}} / \Delta P$ as

$$
\begin{aligned}
\Delta C_{\mathrm{ac}} / \Delta P & =\frac{\Delta C_{\mathrm{ap}} / \Delta P(134 \cdot(4.5+134)-4.5 \cdot 134)}{(4.5+134)^{2}} \\
& \approx 0.9 \frac{\Delta C_{\mathrm{ap}}}{\Delta P}
\end{aligned}
$$

The measurements of $\Delta C_{\mathrm{ac}} / \Delta P$ and $\Delta C_{\mathrm{cp}} / \Delta P$ in $(5 \cdot 8)$ are made using CP-10 circuit. $\Delta V_{\mathrm{ac}} / \Delta P \approx 0.9 \Delta V_{\mathrm{ap}} / \Delta P$ was found in the measurements

Also shown in Fig. 6, the pressure sensitivity of $V_{\text {ab-c }}\left(\Delta V_{\text {ab-c }}\right)$ is $10.4 \mathrm{mV} / \mathrm{kPa}$, which is less than $\Delta V_{\mathrm{ac}} / \Delta P$ value of $11.8 \mathrm{mV} / \mathrm{kPa}$ by $10 \%$. In the $V_{\mathrm{ab}-\mathrm{c}}$ measurements, the $C_{\mathrm{bc}}$ is used as $C_{\mathrm{o}}$ in the CP-10 circuit for offset compensation. As the applied pressure increases, the edge reference capacitance $C_{\mathrm{bc}}$ would increase slightly $\left(\Delta C_{\mathrm{bc}} / \Delta P=0.003 \mathrm{pF} / \mathrm{kPa}\right.$ in the pressure range of $170-280 \mathrm{kPa}$ ) and therefore cause a slight increase in the "offset voltage" $\left(=V_{\mathrm{o}} \cdot C_{\mathrm{bc}} / C_{\mathrm{f}}\right)$. This increase will result in the reduction of the pressure sensitivity $\Delta V_{\mathrm{ab}-\mathrm{c}} / \Delta P$ as

$$
\begin{aligned}
\frac{\Delta V_{\mathrm{ab}-\mathrm{c}}}{\Delta P} & =\frac{V_{\mathrm{g}} \cdot\left(\Delta C_{\mathrm{ac}} / \Delta P\right)-V_{\mathrm{o}} \cdot\left(\Delta C_{\mathrm{bc}} / \Delta P\right)}{C_{\mathrm{f}}} \\
& =\frac{\Delta V_{\mathrm{ac}}}{\Delta P}\left[1-\frac{V_{\mathrm{o}} \cdot\left(\Delta C_{\mathrm{bc}} / \Delta P\right)}{V_{\mathrm{g}} \cdot\left(\Delta C_{\mathrm{ac}} / \Delta P\right)}\right] \\
& =\frac{\Delta V_{\mathrm{ac}}}{\Delta P}\left(1-\frac{2.730 \times 0.003}{2.700 \times 0.030}\right) \cong 0.9 \frac{\Delta V_{\mathrm{ac}}}{\Delta P}
\end{aligned}
$$

For the same reason, $\Delta V_{\text {ab-p }} / \Delta P$ would be equal to $0.9 \Delta V_{\text {ap }} / \Delta P$. The experimental results confirm this as the measured value of $\Delta V_{\mathrm{ab}-\mathrm{p}} / \Delta P$ is $11.8 \mathrm{mV} / \mathrm{kPa}$ and the value of $\Delta V_{\mathrm{ap}} / \Delta P$ is $13.3 \mathrm{mV} / \mathrm{kPa}$. According to the explanations mentioned above, we could have the calculated relationship between $\Delta V_{\text {ab-c }} / \Delta P$ and $\Delta V_{\text {ab-p }} / \Delta P$ as

$$
\begin{aligned}
\Delta V_{\mathrm{ab}-\mathrm{c}} / \Delta P & =0.9\left(\Delta V_{\mathrm{ac}} / \Delta P\right) \approx 0.9\left[0.9\left(\Delta V_{\mathrm{ap}} / \Delta P\right)\right] \\
& \approx 0.9\left\{0.9\left[\left(\Delta V_{\mathrm{ap}-\mathrm{p}} / \Delta P\right) / 0.9\right]\right\} \\
& \approx 0.9 \Delta V_{\mathrm{ab}-\mathrm{p}} / \Delta P
\end{aligned}
$$

The measured values of $\Delta V_{\mathrm{ab}-\mathrm{c}} / \Delta P$ and $\Delta V_{\text {ab-p }} / \Delta P$ are 10.4 and $11.8 \mathrm{mV} / \mathrm{kPa}$, respectively. The experimental result is therefore consistent with the calculated one. This $10 \%$ reduction of the pressure sensitivity of $\Delta V_{\text {ab-c }}$ makes the planar fabrication process possible. The second one is that the use of $C_{\mathrm{bc}}$ as reference capacitor $C_{\mathrm{o}}$ in different applications eliminates the need for choosing different $C_{\mathrm{o}}$ for different stray capacitance external connections. The third one is that these two capacitors located in the same cavity can be used as a differential set to compensate for the common mode environmental changes.
Table 1. The capacitance and voltage outputs of sensor

\begin{tabular}{lllll}
\hline & $C_{\mathrm{ac}}(\mathrm{pF})$ & $C_{\mathrm{bc}}(\mathrm{pF})$ & $V_{\mathrm{ac}}(\mathrm{V})$ & $V_{\mathrm{ab}-\mathrm{c}}(\mathrm{V})$ \\
\hline $25^{\circ} \mathrm{C}$ & 11.444 & 11.662 & 1.396 & 1.353 \\
$60^{\circ} \mathrm{C}$ & 11.505 & 11.825 & 1.425 & 1.350 \\
\hline
\end{tabular}

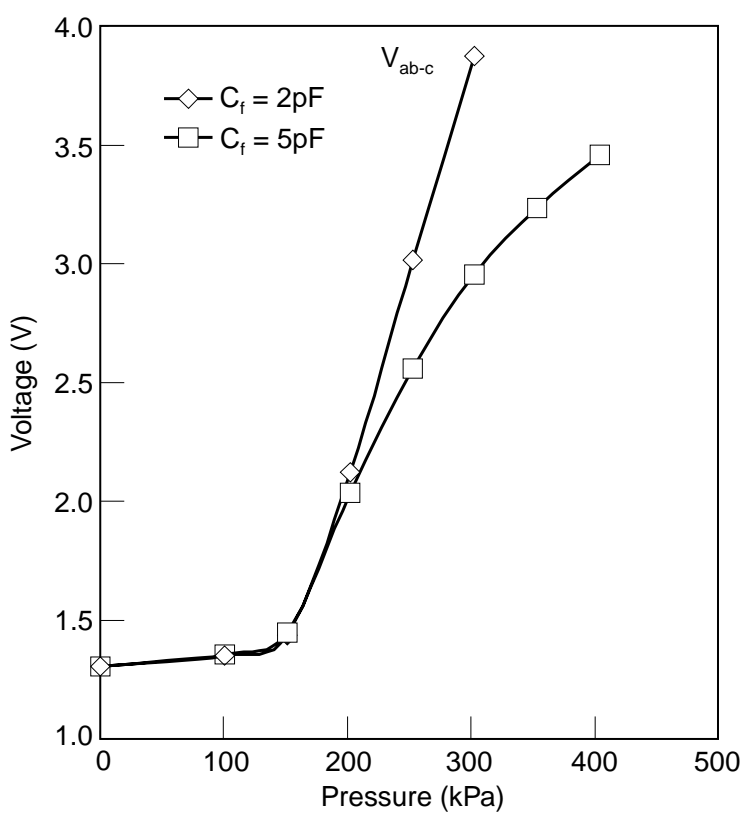

Fig. 7. The voltage-to-pressure characteristics of the sensor

Table 1 lists the measured capacitance and voltage outputs at zero differential pressure at two different temperatures 25 and $60^{\circ} \mathrm{C}$. The capacitances of $C_{\mathrm{ac}}$ and $C_{\mathrm{bc}}$ in the same cavity of sensor increase with increasing temperature. This increase in capacitance due to temperature change would cause an increase of $V_{\mathrm{c}}$ output by $29 \mathrm{mV}$. Meanwhile, the $V_{\mathrm{ab}-\mathrm{c}}$ output is changed only by $3 \mathrm{mV}$ due to the effect that $V_{\mathrm{ab}-\mathrm{c}}$ measurements using the $C_{\mathrm{ac}}$ and $C_{\mathrm{bc}}$ as a differential set could partially cancel out the common mode temperature variations.

The voltage sensitivity can be programmed by the circuit sensitivity. The circuit sensitivity is adjusted by changing either the $V_{\mathrm{g}}$ or $C_{\mathrm{f}}$. Figure 7 shows the increase of the pressure sensitivity of $V_{\text {ab-c }}$ as reducing the external capacitor $C_{\mathrm{f}}$ from $5 \mathrm{pF}$ to $2 \mathrm{pF}$. The stray capacitance existing between the $C_{\mathrm{f}}$ terminals on the CP-10 circuit board is checked to be $4 \mathrm{pF}$. This stray capacitance is in parallel with the $C_{\mathrm{f}}$ connected externally. Therefore, the measured $\Delta V_{\text {ab-c }} / \Delta P$ is increased from 10.4 to $17.0 \mathrm{mV} / \mathrm{kPa}$ as the equivalent capacitance $C_{\mathrm{f}}$ is reduced from 9 to $6 \mathrm{pF}$.

6

\section{Conclusion}

A three-electrode capacitive pressure sensor for touch-mode operation in the pressure range from 170 to $280 \mathrm{kPa}$ is designed and tested. A special ring structure is used to integrate a sensing capacitor and a reference capacitor into the same sensor cavity to partially cancel out the temperature 
effects. This structure offers the advantages of simple fabrication process, planar connections, as well as high sensitivity, improved linear output, and large over range pressure. A third electrode (c) is included to avoid two-level connections without sacrificing its sensitivity. The sensing capacitor $C_{\mathrm{ac}}$ has a pressure sensitivity of $0.030 \mathrm{pF} / \mathrm{kPa}$ in the pressure range from 170 to $280 \mathrm{kPa}$, while the reference capacitor $C_{\mathrm{bc}}$ is insensitive to the applied pressure as expected. These two capacitors $C_{\mathrm{ac}}$ and $C_{\mathrm{bc}}$ are fed as differential inputs to a CP-10 capacitance-to-voltage converter for pressure-tovoltage measurements in order to partially compensate the temperature variation as well as to reduce the stray capacitance effect in the test system and sensor structure. The pressure sensitivity of sensor obtained from the CP-10 is $10.4 \mathrm{mV} / \mathrm{kPa}$ for a given fixed circuit sensitivity $(g)$. The circuit sensitivity can be adjusted by changing the value of $C_{\mathrm{f}}$, when $C_{\mathrm{f}}$ is reduced by a factor $(n)$, the circuit sensitivity will be increased by the same factor $\left(g^{\prime}=n g\right)$. The experimental data show that the sensor sensitivity is increased by a factor of 1.6 (from 10.4 to $17.0 \mathrm{mV} / \mathrm{kPa}$ ) as the $C_{\mathrm{f}}$ is reduced by a factor 1.5 (from 9 to $6 \mathrm{pF}$ ). It is expected that future integration of the sensor and the CP-10 circuit into a single chip could further improve the sensitivity.

\section{References}

Hanneborg A; Ohlckers P (1990) A capacitive silicon pressure sensor with low TCO and high long-term stability. Sensors and Actuators A21-A23: 151-154

Puers B; Peeters E; Bossche A Van den; Sansen W (1990) A capacitive pressure sensor with low impedance output and active suppression of parasitic effects. Sensors and Actuators A21-A23: 108-114

Rosengren L; Soderkvist J; Smith L (1992) Micromachined sensor structures with linear capacitive response. Sensors and Actuators A31: 200-205

Ding X; Li-Jun T; He W; Hau J-T; K Wen H (1990) Touch mode silicon capacitive pressure sensor. 1990 ASME Annual Meeting, Dallax Tx. 11/26/90, DSG-vol 19: 111-117

Ko WH; Wang Q; Wang Y (1996) Touch mode capacitive pressure sensors for industrial applications. Technical Digest of Solid State Sensor and Actuators Workshops, Hilton Head, June 2-8, 1996: 224-228; Ko WH (1996) U.S. Patent No 5585311

Pons P; Blasquez G; Behocaray R (1993) Feasibility of capacitive pressure sensors without compensation circuits. Sensors and Actuators A 37-38: 112-115

Ko Wen H; Bao M-H; Hong Y-D (1982) A high-sensitivity integrated-circuit capacitive pressure transducer. IEEE Trans Electron Dev ED-29 (1): 48-56

Schnatz FV et al. (1992) Smart CMOS capacitive pressure transducer with on-chip calibration capability. Sensors and Actuators A 34: 77-83

Chang K-M; Hwang G-J; Hsieh Y-L; Chen C-H (1998) An accurate determination of the $\mathrm{P}^{+}$silicon layers for microstructure. J Chinese Institute of Electrical Eng 5(2)

Seidel H; Csepregi L; Heuberger A; Baumgratel H (1990) Anisotropic etching of crystalline silicon on alkaline solution. J Electrochem Soc 137 (11): 3626-3632

Ko WH; Yeh GJ (1994) An integrated interfacing circuit for capacitive sensors. Microsystem Technol 1: 42-47 